\title{
Multi-Attribute Decision Making Model with Fuzzy Graph and Its Characteristics
}

\author{
G.Nirmala $^{1} \&$ N.Vanitha ${ }^{2}$ \\ 1. Head \& Associate Professor of Mathematics, Kunthavai Naachiyaar Govt.Arts College for women(Auto.), \\ Thanjavur - 613007. \\ 2. Lecturer of Mathematics, Kunthavai Naachiyaar Govt.Arts College for women(Auto.)Thanjavur - 613007.
}

\begin{abstract}
Various risks and uncertainties exist in construction projects. These may not only prevent the projects to be completed within budget and time limit, but also threaten the quality, safety and operational needs. In making decisions involving multiple objects we have to consider conflicting goals and weigh them against each other. Fuzzy decisions making is a suitable method used by decision makers in uncertain situations in this work. A case study is used to demonstrate the concept of general contractor choice on the basis of multiple attributes of efficiency with fuzzy inputs applying convexity proportional assessment graph method.
\end{abstract}

Keywords: Fuzzy graph, multi-attribute, decision-making model, Fuzzy number

\section{Introduction:}

Decision making "itself, however is broadly defined to include any choice or selection of alternatives, and is therefore of importance in many fields in both the soft social sciences and the hard disciplines of natural sciences and engineering.

Classical decision making generally deals with a set of alternative states of nature a set of alternative actions that are available to the decision maker a relation indicating the state or outcome to be expected from each alternative action and a utility or objective function, which orders the outcomes according to their desirability.

A decision is made under conditions of risk, on the otherhand, when the only available knowledge concerning the outcomes consists of their conditional probability distributions, one for each action. In this case the decision making problem becomes an optimization problem of maximizing the expected utility.

Multiple attribute decision making problems are encountered under various situations where a number of alternatives and actions or candidates need to be chosen based on a set of attributes when we consider a discrete set of alternatives described by some goals. Determine the optimal alternative with the highest degree of desirability with respect to all relevant goals.

Algorithm for multiple attributes decision-making

Establishing system evaluation attributes that relate system capabilities to goals.

Developing alternative systems for attaining the goals(generating alternatives)

Evaluating alternatives interms of attributes (the values of the attributes functions)

Applying a normative multiple attributes analysis method

Accepting one alternative as "optimal"

If the final solutions is not accepted, gather new information and go into the next iteration of multiple attributes optimization.

Multiple attributes decision provides several powerful and effective tools for confronting sorting problems. Comparing the alternatives is the key of making the decision. However in case of conflicting alternatives, a decision maker must also consider imprecise or ambiguous data, which is norm in this type of decision making problems.

\section{Fuzzy Graph And Fuzzy Relational Analysis In Decision Making}

A fuzzy number is an generalization of a regular, real number in the sense that is does not refer to one single value but rather to a connected set of possible values, where each possible value has its own weight between $o$ and 1. This weight is called the membership function. A fuzzy number is a convex, normalized fuzzy set $\tilde{A} \subseteq \mathfrak{R}$ whose membership function is atleast segmentally continuous and has the functional value $\mu_{\widetilde{A}}(x)=1$ at precisely one element.

The fuzzy node fuzzy graph $G$ is defined by the $G=(V, E), \quad V=\left\{v_{i}\left(u_{i}\right)\right\}, E=\left\{e_{i j}\right\}, 0 \leq e_{i j} \leq 1$ 
Where $u_{i}$ is the fuzziness of the node $v_{i}, e_{i j}$ is the fuzziness of the arc from the node $v_{i}$ to the node $v_{j}$.

\section{EXAMPLE:}

The fuzzy node fuzzy graph $G=(V, E)$ is illustrated in fig -1 $V=\left\{v_{1}(0.32), v_{2}(0.43), v_{3}(0.38)\right\}$

$$
E=v_{2} v_{3}\left[\begin{array}{ccc}
v_{1} & v_{2} & v_{3} \\
1 & 0.48 & 0.12 \\
0.74 & 1 & 0.29 \\
0.20 & 0.81 & 1
\end{array}\right]
$$

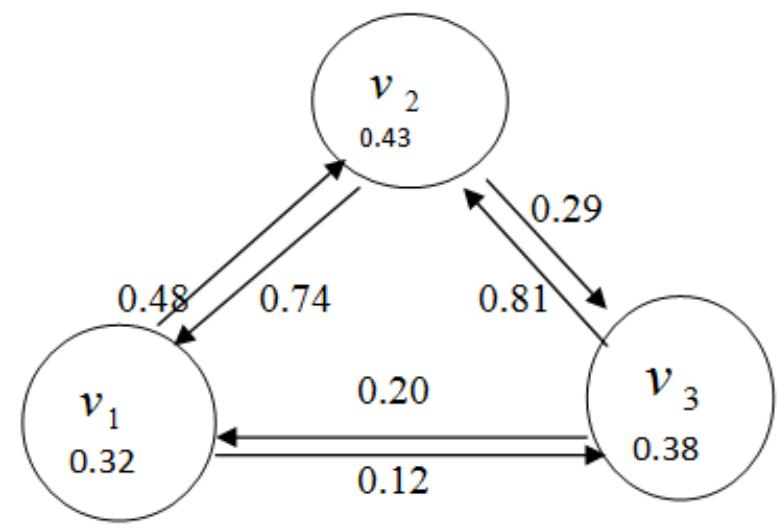

Let $G=(V, E)$ be a fuzzy graph. The degree of a vertex $u$ is $d_{G}(u)=\sum_{u \neq v} E(u v)$. Since

$E(u v)>0 \quad$ for $u v \in E$ and $E(u v)=0$ for $u v \notin E$, this is equivalent to $d_{G}(u)=\sum_{u \neq v} E(u v)$.

Minimum degree of $G$ is $\delta(G)=\wedge\{d(v) / v \in V\}$

Maximum degree of $\mathrm{G}$ is $\Delta(G)=\vee\{d(v) / v \in V\}$.

Fuzzy convex is the membership grade starts at zero, rises to a maximum and then declines to zero again as the domain increases. This method is useful for value evaluation of maximizing as well as minimizing criteria. Ranking alternatives by the convexity method assumes direct and proportional dependence of significance and priority of investigated alternatives on a system of criteria. The determination of significance and priority of alternatives, by using convexity proportional assessment in fuzzy graph method. The presented procedure of that method indicated that it can be easily applied for evaluating the alternatives and selecting the most efficient one, with decision maker being completely aware of the physical meaning of the process.

\section{Problem :}

The set of attributes and initial values of attributes are determined on the basis of expert, normative and calculation methods. The set of alternatives $X=\left\{x_{1}, x_{2}, x_{3}\right\}$ and the goals be given as

$$
\begin{aligned}
& G_{1}\left(x_{i}\right)=\left\{\left(x_{1}, 0.8\right),\left(x_{2}, 0.4\right),\left(x_{3}, 0.6\right)\right\} \\
& G_{2}\left(x_{i}\right)=\left\{\left(x_{1}, 0.6\right),\left(x_{2}, 0.6\right),\left(x_{3}, 0.8\right)\right\} \\
& G_{3}\left(x_{i}\right)=\left\{\left(x_{1}, 0.4\right),\left(x_{2}, 0.6\right),\left(x_{3}, 0.8\right)\right\} \\
& G_{4}\left(x_{i}\right)=\left\{\left(x_{1}, 0.2\right),\left(x_{2}, 0.8\right),\left(x_{3}, 0.6\right)\right\}
\end{aligned}
$$

The weights of the goals have been established as $\left(q_{1}, q_{2}, q_{3}, q_{4}\right)=(0.28,0.16,0.32,0.24)$ then we find the optimal alternative using by convexity proportional assessment in graph method.

\section{Solution:}

Given the decision making graph $G$ is denoted by 


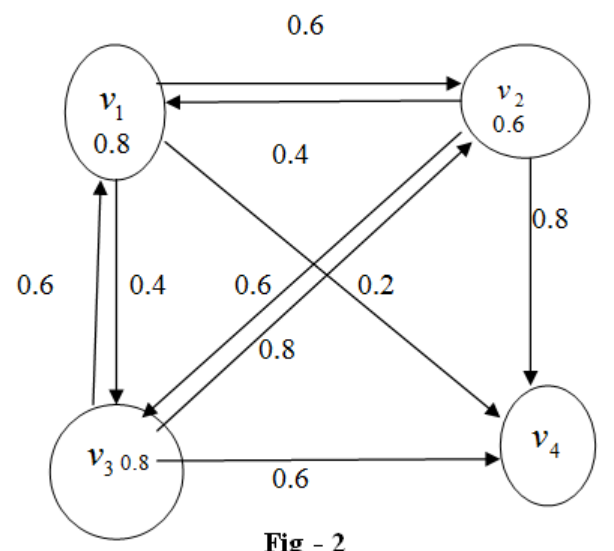

Fig - 2

Normalization of the decision making graph $\bar{G}$. The normalized values of this graph are calculated as

$$
\begin{array}{rll}
\bar{x}_{j i}=\frac{x_{j i}}{\sum_{j=1}^{n} x_{j i}}, \quad j=1 \text { to } 3 \text { and } i=1 \text { to } 4 & \\
\bar{x}_{11}=0.44 & \bar{x}_{21}=0.22 & \bar{x}_{31}=0.33 \\
\bar{x}_{12}=0.3 & \bar{x}_{22}=0.3 & \bar{x}_{32}=0.4 \\
\bar{x}_{13}=0.22 & \bar{x}_{23}=0.33 & \bar{x}_{33}=0.44 \\
\bar{x}_{14}=0.13 & \bar{x}_{24}=0.5 & \bar{x}_{34}=0.38
\end{array}
$$

Normalized decision making graph becomes

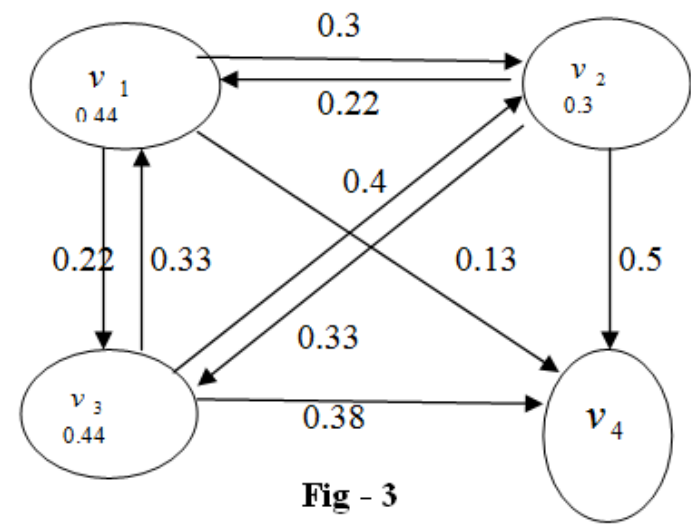

Calculation of the weighted normalized decision making graph $\hat{G}$. The weighted normalized values $\hat{x}_{i j}$ are calculated as

$\hat{x}_{i j}=\bar{x}_{i j} \cdot q_{i}, \quad j=1$ to 3 and $i=1$ to 4

Where $q_{i}$ is weight of the $\mathrm{i}^{\text {th }}$ attribute

$$
\begin{array}{lll}
\hat{x}_{11}=0.12 & \hat{x}_{21}=0.06 & \hat{x}_{31}=0.09 \\
\hat{x}_{12}=0.05 & \hat{x}_{22}=0.05 & \hat{x}_{32}=0.06 \\
\hat{x}_{13}=0.07 & \hat{x}_{23}=0.11 & \hat{x}_{33}=0.14 \\
\hat{x}_{14}=0.03 & \hat{x}_{24}=0.12 & \hat{x}_{34}=0.09
\end{array}
$$

Weighted normalized decision making graph becomes 


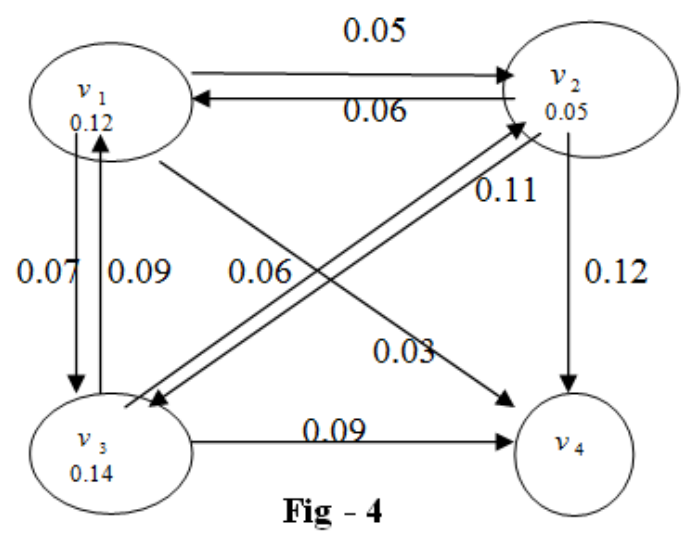

Sums $\boldsymbol{P}_{j}$ of attributes values which larger values are more preferable. That is adding adjacent node

from $v_{1}, v_{2}, v_{3}$ we get $\left(P_{1}, P_{2}, P_{3}\right)=(0.27,0.34,0.38)$.

Now $k$ is number of attributes which must to be maximized, that is $\boldsymbol{k}=3$

Sums $\boldsymbol{R}_{j}$ of attributes values which smaller values are more preferable.

Here $\left(\begin{array}{lll}\boldsymbol{R}_{1} & \boldsymbol{R}_{2} & \boldsymbol{R}_{3}\end{array}\right)=(0.03,0.12,0.09)$

Determining the minimal value of $\boldsymbol{R}_{j}$

$$
\begin{aligned}
& R_{\text {min }}=\min _{j} R_{j} \quad j=1 \text { to } 3 \\
& R_{\text {min }}=R_{1}=0.03
\end{aligned}
$$

Calculation of the relative weight of each alternative

$$
\begin{aligned}
& Q_{j}=P_{j}+\frac{\sum_{j=1}^{n} R_{j}}{R_{j} \sum_{j=1}^{n} \frac{1}{R_{j}}} \\
& \left(Q_{1}, Q_{2}, Q_{3}\right)=(0.42,0.38,0.43) \\
& K=\max _{j}\{0.42,0.38,0.43\}
\end{aligned}
$$

Calculation of the utility degree of each alternative $N_{j}=\frac{Q_{j}}{Q_{\max }} 100 \%$

The utility degree of each alternative is $\left(N_{1}, N_{2}, N_{3}\right)=(98 \%, 88 \%, 100 \%)$.

Thus the best third alternative is selected according to convexity proportional assessment in graph method. The corresponding alternative rank as follows $x_{3}>x_{1}>x_{2}$.

\section{Conclusions}

In real life multi-attribute modeling of multi alternative assessment problems attributes values, which deals with the future, can to be expressed in fuzzy numbers. Convexity proportional assessment is developed in fuzzy graph for assessment of alternatives by multiple attribute values determined in fuzzy number. This approach is intended to support the decision making process and increase the efficiency of the resolution process. Convexity proportional assessment method can be applied to the solution of ranking alternatives in fuzzy graph. 


\section{References}

[1]. Zavadskas,E.K., A.Zakarevicius and J.Antuchevi cien e(2006).Evaluation of ranking accuracy in multi-criteria decisions. Informatica, 17(4), $601-618$

[2]. Zavadskas, E.K., A.Kaklauskas, F.Peldschus and Z.Turskis(2007b).Multi-attribute assessment of road design solutions by using the COPRAS method. The Baltic Journal of Road and Bridge Engineering,2(4), $195-203$

[3]. G.Nirmala and N.Vanitha, Risk of Construction project with Fuzzy Characteristics, Narosa Publication, 2010

[4]. G.Nirmala and N.Vanitha, Fuzzy Graph in Geology, International Journal of Scientific Research, May 2013, ISSN No. 2277-8179

[5]. G.Nirmala and N.Vanitha, Risk analysis of river- type hydropower plants, Aryabhatta Journal of Mathematics and informatics, May 2014, Volume I

[6]. G.Nirmala and N.Vanitha, Applications of Fuzzy decision making for the stable Marriage Problem, The PMU Journal of Humanities and Sciences, 2012

[7]. G.Nirmala and K.Uma, Maximal Bipartite Fuzzy Graph Algorithm, Narosa Publication,2010

[8]. G.Nirmala and K.Uma, Fuzzy Graph - Estimations of strongest weight, The PMU Journal of Humanities and Sciences.

[9]. G.Nirmala and N.Vanitha, Decision-Making Model by Applying Fuzzy Numbers in Construction Project, International Journal of Scientific Research Publication,June 14,2014. 